\title{
472167 - PREHOSPITAL VS HOSPITAL AIRWAY MANAGEMENT IN TRAUMA PATIENTS
}

\author{
Shawn Hicks, MD, MSc, David Neilipovitz, MD, Greg Bryson, MD \\ Anesthesia, University of Ottawa, Ottawa, ON, Canada
}

Introduction: There is disagreement in the literature regarding the benefit of invasive prehospital airway management in the trauma patient. Retrospective studies of traumatic brain injury (TBI) have suggested that out of hospital endotracheal intubation (ETI) increases the risk of adverse outcome over ETI in the emergency department (1). One randomized controlled trial in the pediatric population demonstrated prehospital ETI did not improve survival or neurologic outcome (2). In this study we were interested in examining the effect of prehospital ETI on outcome in our trauma patients.

Methods: After obtaining approval from our research ethics board, we conducted a retrospective chart review on all of our trauma patients from 2000-05. Our trauma service maintains an electronic database of all trauma patients in our tertiary care hospital system. The database includes demographics, type of injury, location of injury, abbreviated injury score (AIS), paramedic call sheet data, emergency department treatment, course in hospital, functional assessment at discharge and follow up, length of stay and mortality. AIS is a calculated score used to assess overall injury based on the 3 most severely injured body regions. Cases were included if an adult trauma patient needed ETI in the emergency department or if ETI was attempted by paramedics in the field. We included all trauma patients, not only those with isolated TBI. Our primary outcome measure was mortality.

Results: The database for the 5 yr period included 2977 trauma patients. A total of 251 cases were included in the final analysis, $71 \%$ of these patients were transferred from peripheral hospitals. Patients were intubated by paramedics in $24.8 \%$ of cases, $10 \%$ by physicians at the Ottawa hospital and $63.7 \%$ at peripheral hospitals. The overall mortality in the ETI group was $38.2 \%$ for the study period. Mortality was higher in patients who had ETI performed by paramedics, 55\% compared to 31\% ( $<<0.0001)$. Patients who had ETI attempted by paramedics were more severely injured, AIS of 34.9 vs. 29.13, ( $\mathrm{F}=16.4$, $\mathrm{p}<0.0001$ ). A total of $72 \mathrm{ETI}$ attempts were made by paramedics, $84.7 \%$ success rate. Patients transferred to our hospital from a peripheral hospital had a mortality of $29.2 \%$ compared to $61.4 \%$ in patients brought directly to our hospital ( $<<0.0001$ Chi2). Length of stay in hospital was similar between groups. Logistic regression analysis applied to this data revealed increased mortality for a higher AIS score and for higher age, but no effect for attempted ETI by paramedics or for gender.

Discussion: These results suggest that patients who undergo an attempt at ETI by paramedics have a higher mortality but are also more severely injured. Our findings are difficult to compare to previous work in this area that focused on isolated TBI. This study was also limited by a survival bias in the group of patients transferred to our center from other hospitals. Our database does not capture those patients who were not transferred to our hospital because they died in a referring hospital.

References: (1)Wang 2004 Ann Emerg Med. 44:439-50 (2)Gausche 2000 JAMA 
283:738-90.

ETI attempted by EMS and mortality

\begin{tabular}{|c|c|c|c|}
\hline & Alive & Dead & Total \\
\hline EMS & $32(44.4 \%)$ & $40(55.6 \%)$ & 72 \\
\hline MD & $119(68.8 \%)$ & $54(31.2 \%)$ & 173 \\
\hline Total & 151 & 94 & 245 \\
\hline
\end{tabular}

$\mathrm{p}<0.0001$ Chi-square 